\title{
A polling system whose stability region depends on a whole distribution of service times
}

\author{
Natalia Chernova $\quad$ Sergey Foss $\quad$ Bara Kim
}

\begin{abstract}
We present an example of a single-server polling system with two queues and an adaptive service policy where the stability region depends on the expected values of all the primitives and also on a certain exponential moment of the service-time distribution in one of the queues. The latter parameter can not be determined, in general, in terms of any finite number of power moments. It follows that the fluid approximation approach may not be an appropriate tool for the stability study of this model.

Keywords: Polling System, Adaptive Limited and Exhaustive Service Disciplines, Stability Conditions, Foster Criterion.

Subject Classifications: 60K25, 68M20
\end{abstract}

\section{Introduction}

We deal with a class of cyclic polling systems which are single-server systems where the server visits a finite number of queues in a cyclic order and serves customers there. The stability and performance analysis of polling models has been a hot topic in the last 15-20 years. For overviews on the current progress in the studies of polling models, see, for example, Borst (1995); Boxma et al. (2009); Wierman et al. (2007); Winands et al. (2009); Boon (2011). Stability conditions in many polling models and, in particular, in those with state-independent service policies may be analysed via the fluid approximation approach (see Rybko and Stolyar (1992) and Dai (1995)) that is based on the Functional Strong Law of Large Numbers and involves only the first moments of driving random elements. However, it is known (may be, not sufficiently broadly) that, in general, a dependence of stability conditions on distributions (of stochastic characteristics of polling models) may be more complex and may involve into consideration not only power moments but whole primitive distributions.

In this article, we present a simple and natural example of a polling system with an adaptive service mechanism. More precisely, we introduce a 2-station system with an exhaustive service policy at one of them and a limited policy at the other, where the limited policy increases the limit level if the server finds the other station empty. We assume the input streams to be Poisson, the switch-over times to be exponential, while the service time distributions in both queues are assumed to be rather general.

We show that the stability conditions depend on parameters of the Poisson processes and of the 
exponential random variables, on the first moments of the service time distributions from both stations, and also on an exponential moment (!) of the service time at the station where the limited policy is in use. In particular, this shows that, in general, a knowledge of any finite number of power moments is not enough to determine the stability region. This also shows that the direct probabilistic methods are still of great importance in the stability analysis of stochastic networks and that the fluid approximation approach is not as universal as many researchers think.

This paper may be viewed as complementary to Chernova et al. (2011), where the authors analysed another single-server cyclic polling system with three queues and a similar adaptive rule, and obtained upper and lower bounds for the stability region using the fluid approximation approach. They provided the simulation results showing that stability conditions for systems with the same power moments of primitive random variables (up to the third moment), but with different distributions, may also differ.

\section{The Model and Its Stability Conditions}

We consider a polling system with 2 stations and a single server that consequently visits the stations and serve customers there. Customers arrive at station $k=1,2$ in a Poisson stream of intensity $\lambda_{k}>0$. Service times at station $k$ form an i.i.d. sequence having distribution function $B_{k}$ with positive finite mean $b_{k}$. It takes an exponential time with mean $\gamma$ for the server to travel either from station 1 to station 2 or from station 2 to station 1. We assume all primitive random variables (interarrival, service, and travel times) to be mutually independent. Server follows the exhaustive policy at station 2 and an adaptive limited policy at station 1 . In more detail, the server works in "cycles", and each cycle starts when the server arrives at station 2 .

In more detail, if the server finds at least one customer at station 2 when he arrives there, he starts to serve customers one-by-one (including new arrivals) until he empties the queue. Then he travels to station 1 (during an exponential time), serves one customer there (if there is any) or leaves it immediately (if the queue is empty), and then travels back to station 2 (during another exponential time). This is a standard cycle.

If, upon his arrival to station 2, the server finds it empty, the next cycle is modified: the server is allowed to serve $m$ extra customers at station 1 . More precisely, he starts the cycle with his travel to station 1 ; then he provides a service at station 1 until either he completes $1+m$ services or the station becomes empty (here $m$ is a fixed non-negative integer). Then he travels back to station 2.

We need the cycles to be finite and, moreover, to have a finite mean. For that, we need the mean time to empty the second queue to be finite. So we assume the following condition to hold:

$$
\rho:=\lambda_{2} b_{2}<1
$$

We are interested in stability conditions for this model. For $t \geq 0$, let $Q_{k}(t), k=1,2$, be the queue length at station $k$ at time $t$. Let $L(t)$ be the location of the server. More precisely, $L(t)=k$ if the server is in service at station $k$, and $L(t)=(1,2)$ or $L(t)=(2,1)$ if the server is travelling either from 
1 to 2 or back. Let $R(t)$ be the residual service time if the server is in service (and let $R(t)=0$ if the server is travelling). Let $I(t)$ be 1 if the cycle is standard and 2 if the cycle is modified. Then the vector $Z(t)=\left(Q_{1}(t), Q_{2}(t), L(t), R(t), I(t)\right)$ represents the current state of the system. We assume the process $Z(t)$ to be right-continuous. This is a continuous-time Markov process. We seek for conditions for the distribution of $Z(t)$ to converge weakly, as $t \rightarrow \infty$, to a proper limiting distribution.

\subsection{Embedded Processes}

For $i=1,2$ and $n=1,2, \ldots$, let $Q_{n, i}$ be the number of customers at station $i$ when the server arrives there for the $n$th time. Let $Z_{n}=\left(Q_{n, 1}, Q_{n, 2}\right), n=1,2, \ldots$. Since the input processes are Poisson, the sequence $\left\{Z_{n}\right\}$ forms a time-homogeneous Markov chain taking values in a countable state space $\mathcal{Z}_{+}^{2}$, and any two states from the state space communicate to each other. Since cycle lengths include exponential travel times, their distributions are absolutely continuous, and, in particular, the following conclusion may be deduced (see, e.g., Asmussen (2003) for the background):

Proposition 1. Under the condition (1), the process $\{Z(t): t \geq 0\}$ has a proper limiting distribution (is stable) if and only if the Markov Chain $\left\{Z_{n}: n=1,2, \ldots\right\}$ is positive recurrent.

\subsection{Auxiliary Markov Chain}

In this subsection, we assume that initially, at time $t=0$, there is infinitely many customers at station 1 and no customers at station 2. Assume also that, at time 0 , the server starts to travel from station 2 to station 1 . For $t \geq 0$, let $\hat{Z}(t)$ be the number of customers at station 2 at time $t$ (so $\hat{Z}(0)=0$. Then $\{\hat{Z}(t): t \geq 0\}$ is a regenerative process, and a regeneration occurs when the server arrives to queue 2 and finds it empty. The first renegerative time is denoted by $T>0$.

For $n=1,2, \ldots$, let $t_{n}$ be the $n$th return time of the server to station 2 , so $t_{0}:=0$. Let $\hat{Q}_{n}:=\hat{Z}\left(t_{n}\right)$, $n=0,1,2, \ldots$ Then $\left\{\hat{Q}_{n}: n=0,1,2, \ldots\right\}$ forms a Markov chain. Moreover, random sequence $X_{n}:=$ $\mathbf{I}\left(\hat{Q}_{n}>0\right)$ is also Markov. It takes only two values: 0 if the queue is empty, and 1, otherwise. Indeed, if $X_{n}=0$, then $X_{n+1}=0$ if there is no arrivals to station 2 during two travel times (from station 2 to station 1 and back) and $1+m$ service times at station 1 , i.e.,

$$
p_{0,0}:=\mathbf{P}\left(X_{n+1}=0 \mid X_{n}=0\right)=\left(\frac{\gamma^{-1}}{\lambda_{2}+\gamma^{-1}}\right)^{2}\left(B_{1}^{*}\left(\lambda_{2}\right)\right)^{1+m} .
$$

Notice that $\frac{\gamma^{-1}}{\lambda_{2}+\gamma^{-1}}$ is the probability that there is no arrival to station 2 during a travel time of the server, and $B_{1}^{*}\left(\lambda_{2}\right)=\int_{0}^{\infty} e^{-\lambda_{2} t} d B_{1}(t)$ is the probability that there is no arrivals to station 2 during a service time of a customer at station 1 . Clearly

$$
B_{1}^{*}\left(\lambda_{2}\right)=\mathbf{E} e^{-\lambda_{2} \sigma_{1}}
$$

where $\sigma_{1}$ is a generic service time of a customer at station 1. For the Markov chain $\left\{X_{n}\right\}$, the transition probability from 0 to 1 is given by $p_{0,1}:=\mathbf{P}\left(X_{n+1}=1 \mid X_{n}=0\right)=1-p_{0,0}$. If $X_{n}=1$, then, for 
any queue length, the server first empties queue 2. After that, the server travels to station 1 , serves a customer at station 1 , and travels back to station 2. Therefore, given $X_{n}=1$, the probability that the server finds station 2 empty when returns there next time is

$$
p_{1,0}:=\mathbf{P}\left(X_{n+1}=0 \mid X_{n}=1\right)=\left(\frac{\gamma^{-1}}{\lambda_{2}+\gamma^{-1}}\right)^{2} B_{1}^{*}\left(\lambda_{2}\right) .
$$

Finally, the transition probability $p_{1,1}$ is given by $p_{1,1}:=\mathbf{P}\left(X_{n+1}=1 \mid X_{n}=1\right)=1-p_{1,0}$.

Let $\nu$ be the first return time to 0 for the Markov chain $\left\{X_{n}\right\}$,

$$
\nu:=\min \left\{n \geq 1: X_{n}=0\right\} .
$$

Then, by straightforward calculations,

$$
\mathbf{E} \nu=1+\frac{p_{0,1}}{p_{1,0}}
$$

and

$$
T=\sum_{i=1}^{\nu} \psi_{i}
$$

where $\psi_{i}$ is a duration of the $i$ th cycle (in continuous time), which is the sum of the service times at both stations and of the travel times. Hence

$$
\begin{aligned}
\mathbf{E} T & =\sum_{i=1}^{\infty} \mathbf{E}\left(\psi_{i} \mathbf{I}(\nu \geq i)\right) \\
& =\mathbf{E} \psi_{1}+\sum_{i \geq 2} \mathbf{E}\left(\psi_{i} \mathbf{I}(\nu \geq i-1) \mathbf{I}\left(\hat{Q}_{i-1} \geq 1\right)\right) \\
& =\mathbf{E} \psi_{1}+\sum_{i \geq 2}\left(\mathbf{E}\left(\hat{Q}_{i-1} \mid \nu \geq i-1\right) \frac{b_{2}}{1-\rho} \mathbf{P}(\nu \geq i-1)+\left(2 \gamma+b_{1}\right) \mathbf{P}(\nu \geq i)\right),
\end{aligned}
$$

where we have used the fact that, in an $M / G / 1$ queue with input rate $\lambda_{2}$ and mean service time $b_{2}$, the mean of a busy period is $b_{2} /(1-\rho)$. Note that

$$
\mathbf{E} \psi_{1}=2 \gamma+(1+m) b_{1}, \quad \mathbf{E} \hat{Q}_{1}=\left(2 \gamma+(1+m) b_{1}\right) \lambda_{2}, \quad \mathbf{E}\left(\hat{Q}_{i-1} \mid \nu \geq i-1\right)=\left(2 \gamma+b_{1}\right) \lambda_{2},
$$

for any $i \geq 3$. By substituting these expressions, we obtain

$$
\mathbf{E} T=\left(2 \gamma+b_{1}\right) \frac{1}{1-\rho} \mathbf{E} \nu+\frac{1}{1-\rho} m b_{1} .
$$

Since the number of service completions at station 1 before time $T$ is $\nu+m$, the mean service rate at station 1 is given by

$$
r:=\frac{\mathbf{E}[\nu+m]}{\mathbf{E} T}=\frac{(\mathbf{E} \nu+m)(1-\rho)}{\left(2 \gamma+b_{1}\right) \mathbf{E} \nu+m b_{1}} .
$$

\subsection{Stability Analysis}

Based on the calculations from the previous subsection, we obtain the following result.

Theorem 1. The Markov chain $\left\{Z_{n}\right\}$ is positive recurrent if and only if inequality

$$
r>\lambda_{1},
$$

holds. 
Remark. Condition (2) may be equivalently written as

$$
2 \gamma \lambda_{1}+\rho_{0}-1<\frac{\left(1-\rho_{0}\right) m\left(\frac{\gamma^{-1}}{\lambda_{2}+\gamma^{-1}}\right)^{2} B_{1}^{*}\left(\lambda_{2}\right)}{1+\left(\frac{\gamma^{-1}}{\lambda_{2}+\gamma^{-1}}\right)^{2} B_{1}^{*}\left(\lambda_{2}\right)\left[1-\left(B_{1}^{*}\left(\lambda_{2}\right)\right)^{m}\right]},
$$

where $\rho_{0}=\lambda_{1} b_{1}+\lambda_{2} b_{2}$. Clearly, (3) implies $\rho_{0}<1$ and, therefore, implies (10).

Proof is based on the standard Lyapunov arguments. We provide just a short outline of the proof.

Sufficiency. Assume (2) holds. Define $\tau_{k}, k=1,2, \ldots$, by

$$
\tau_{k}=\inf \left\{n>\tau_{k-1}: Q_{n, 2}=0\right\}, \quad k=1,2, \ldots,
$$

with $\tau_{0}=0$. Then

$$
\Delta_{k}:=\mathbf{E}\left(\left(Q_{\tau_{2}, 1}-Q_{\tau_{1}, 1}\right) \mid Q_{\tau_{1}, 1}=k\right) \rightarrow \lambda_{1} \mathbf{E} T-(\mathbf{E} \nu+m)<0,
$$

as $k \rightarrow \infty$. Since $\sup _{k \geq 0} \Delta_{k}$ is finite, the embedded Markov chain $Z_{\tau_{k}}=\left(Q_{\tau_{k}, 1}, Q_{\tau_{k}, 2}\right)=\left(Q_{\tau_{k}, 1}, 0\right)$ is positive recurrent, by the Foster criterion. Then the Markov chain $\left\{Z_{n}\right\}$ is also positive recurrent, since the mean cycle time, $\mathbf{E} \tau_{2}-\mathbf{E} \tau_{1}$, is bounded from above by $\mathbf{E} T<\infty$.

Necessity. If (11) fails, then the mean time to empty the second queue cannot be finite. Assume now that (11) holds and (2) fails. Then the queue lengths at the first station at the embedded moments of server's arrivals there couple, with a positive probability, with consecutive values of a random walk with non-negative drift. So the Markov chain $\left\{Z_{n}\right\}$ cannot be positive recurrent.

We conclude with the following

Remark. From the expression for $\mathbf{E} \nu$, one can easily see that the stability condition (2) depends on the "negative" exponential moment $B_{1}^{*}\left(\lambda_{2}\right)$, unless either

(a) $m=0$, i.e. there is no an adaptive mechanism; then $r=(1-\rho) /\left(2 \gamma+b_{1}\right)$ or

(b) $\gamma=0$, i.e. travel times are zeros; then $r=(1-\rho) / b_{1}$.

The stability conditions in cases (a)-(b) are well-known.

Acknowledgement. SF thanks Onno Boxma for a useful discussion.

The research of N. Chernova was partially supported by the Ministry of Higher Education and Science of the Russian Federation Grant RNP.2.1.1.346. The research of S. Foss was partially supported by the London Mathematical Society travel grant and by the Royal Society International Joint Project. The research of B. Kim was supported by Basic Science Research Program through the National Research Foundation of Korea(NRF) funded by the Ministry of Education, Science and Technology(2011-0004133).

\section{References}

[1] S. Asmussen (2003). Applied Probability and Queues, 2nd Edition. Springer, New York.

[2] M. Boon (2011). Polling Models. From Theory to Traffic Intersections. Proefshrift. Eindhoven University. 
[3] S. C. Borst (1995). Polling systems. CWI tracts, 115. Centrum voor Wiskunde en Informatica, Amsterdam.

[4] O. J. Boxma, J. Bruin, B. H. Fralix (2009). Sojourn times in polling systems with various service disciplines. Perform. Eval., 66, 621-639.

[5] N. Chernova, S. Foss, and B. Kim (2011). On the Stability of a Polling System with an Adaptive Service Mechanism, to appear in Annals of Operations Research.

[6] J. G. Dai (1995). On positive Harris recurrence of multiclass queueing networks: A unified approach via fluid models. Ann. Appl. Probab., 5, 49-77.

[7] A. N. Rybko and A.L. Stolyar (1992). Ergodicity of stochastic processes describing the operation of open queueing networks. Problems of Information Transmission, 28, 3-26.

[8] V. M. Vishnevsky, O. V.Semenova (2008). Adaptive dynamical polling in wireless networks. Cybernetics and Information Technologies, 8, 3-11.

[9] A. Wierman, E. M. M. Winands, O. J. Boxma (2007). Scheduling in polling systems. Perform. Eval., 64, 1009-1028.

[10] E. M. M. Winands, I. J. B. F. Adan, G. J.van Houtum, D. G. Down (2009). A state-dependent polling model with $k$-limited service. Prob. Eng. Inform. Sci., 23, 385-408. 